\title{
Intercomparison of Methods for Determination of Resonant Frequency Shift of a Microstrip Patch Antenna Loaded with Hevea Rubber Latex
}

\author{
Nor Zakiah Yahaya, ${ }^{1}$ Zulkifly Abbas, ${ }^{2,3}$ Borhanuddin Mohd Ali, ${ }^{4}$ \\ Alif Ismail, ${ }^{2}$ and Farizah Ansarudin ${ }^{5}$ \\ ${ }^{1}$ School of Physics, Universiti Sains Malaysia (USM), 11800 Pulau Pinang, Malaysia \\ ${ }^{2}$ Department of Physics, Faculty of Science, Universiti Putra Malaysia (UPM), 43400 Serdang, Selangor Darul Ehsan, Malaysia \\ ${ }^{3}$ Institute for Mathematical Research, Universiti Putra Malaysia (UPM), 43400 Serdang, Selangor Darul Ehsan, Malaysia \\ ${ }^{4}$ Department of Computer and Communications System Engineering, Faculty of Engineering, Universiti Putra Malaysia (UPM), \\ 43400 Serdang, Selangor Darul Ehsan, Malaysia \\ ${ }^{5}$ Department of Electrical, Electronic and Systems Engineering, Faculty of Engineering and Built Environment, \\ Universiti Kebangsaan Malaysia, 43600 Bangi, Selangor, Malaysia
}

Correspondence should be addressed to Zulkifly Abbas; za@upm.edu.my

Received 9 April 2014; Accepted 9 July 2014; Published 3 August 2014

Academic Editor: Ignacio R. Matias

Copyright (C) 2014 Nor Zakiah Yahaya et al. This is an open access article distributed under the Creative Commons Attribution License, which permits unrestricted use, distribution, and reproduction in any medium, provided the original work is properly cited.

\begin{abstract}
This paper presents an intercomparison between the finite element method, method of moment, and the variational method to determine the effect of moisture content on the resonant frequency shift of a microstrip patch loaded with wet material. The samples selected for this study were Hevea rubber latex with different percentages of moisture content from $35 \%$ to $85 \%$. The results were compared with the measurement data in the frequency range between $1 \mathrm{GHz}$ and $4 \mathrm{GHz}$. It was found that the finite element method is the most accurate among all the three computational techniques with 0.1 mean error when compared to the measured resonant frequency shift. A calibration equation was obtained to predict moisture content from the measured frequency shift with an accuracy of $2 \%$.
\end{abstract}

\section{Introduction}

In recent years, microstrip patch antenna has been gaining popularity as a new method to determine complex permittivity of materials [1-4]. The microstrip was supported on a substrate material and covered with the material under test. The microstrip fringing field interacts with the sample and produces a change in its effective impedance which is measurable in terms of the shift of the resonant frequency and reflection coefficient which can be calibrated in terms of moisture for a "wet" sample of relatively high permittivity. Unfortunately, theoretical calculations for the impedance were only reported using the variational expressions for the line capacitance $[5,6]$.
In this paper, in addition to the Variational method, both the finite element method (FEM) and method of moment (MoM) were also used to calculate the reflection coefficient, $\Gamma$ of the microstrip patch sensor designed to operate in the frequency between $1 \mathrm{GHz}$ and $4 \mathrm{GHz}$. The variation in the resonant frequency shift, $f_{\text {shift }}$ with moisture content using the three methods were compared with the measurement data. The samples used in this work were Hevea rubber latex with different percentages of moisture content (m.c.) ranging from $35 \%$ to $85 \%$. Different percentages of moisture will give different values of both the dielectric constant and loss factor at various frequencies which in turn will affect the effective impedance of the patch antenna and thus the resonant frequency of the sensor. 


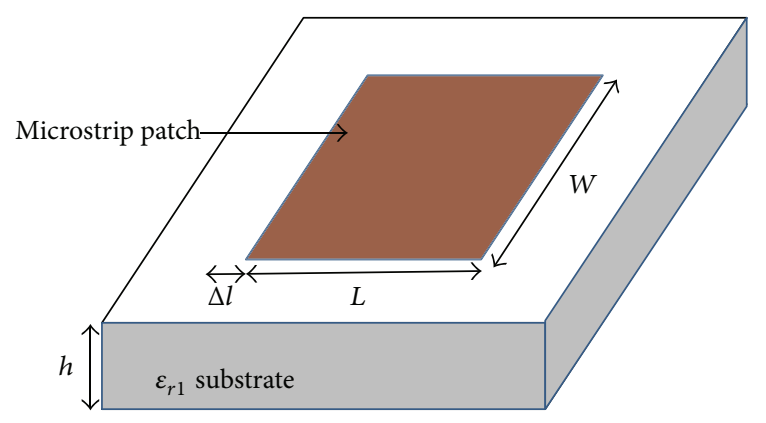

FIGURE 1: Microstrip rectangular geometry.

\section{Formulation}

2.1. Reflection Coefficient. The reflection coefficient of the material under test can be calculated as follows:

$$
\Gamma_{s}=\left|\frac{Z_{s}-Z_{0}}{Z_{s}+Z_{0}}\right|
$$

where $Z_{0}$ and $Z_{s}$ are the characteristic impedance of the unloaded and loaded sensors.

2.1.1. Variational Technique. The calculation of reflection coefficient of a microstrip covered with wet samples using variational method [4] can be found by defining the characteristic impedance

$$
Z_{s}=\frac{1}{c \sqrt{C_{a} C}}
$$

where $c$ is the free-space speed of light and capacitance, $C$ can be written

$$
\begin{aligned}
\frac{1}{C}= & \frac{1}{2 \pi Q^{2}} \\
& \times \frac{[\tilde{f}(\beta)]^{2} d(\beta h)}{\left[\varepsilon_{r 1}\left(\varepsilon_{r 1 \tanh \beta d+1} / \varepsilon_{r 1+\tanh \beta d}\right)+\varepsilon_{r 2} \operatorname{coth}(\beta h)\right](\beta h)},
\end{aligned}
$$

where $\tilde{f}$ is the Fourier Transforms of charge distribution, $\beta$ is the Fourier Transform variable, $h$ is the substrate, thickness, $d$ is the thickness of sheet material covering the microstrip, $\varepsilon_{r 1}$ and $\varepsilon_{r 2}$ are the relative permittivities of the substrates and wet sample, respectively (see Figure 2 ). $Q$ denotes the total charge on the strip conductor, and $C_{a}$ is the capacitance per unit length of the structure with the dielectrics replaced by air.

For the rectangular (or square) geometry (patch antenna) shown in Figure 1, the lowest resonant frequency, $f_{r}$, can be determined [5], once the effective dielectric constant of the covered microstrip is known

$$
f_{r}=\frac{c}{2(L+2 \Delta l) \sqrt{\varepsilon_{\text {eff }}}}
$$

$\varepsilon_{r 3}$ air

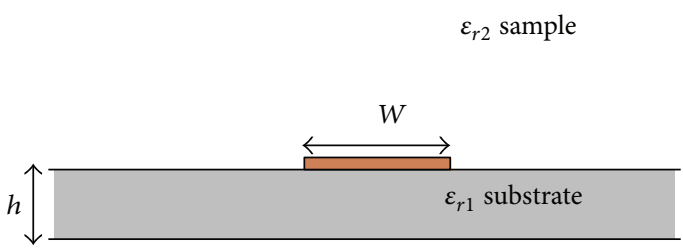

FIGURE 2: Microstrip configuration.

$c=3 \times 10^{8} \mathrm{~m} / \mathrm{s}$

$$
\Delta l=0.412 h \frac{\left(\varepsilon_{\mathrm{eff}}+0.3\right)}{\left(\varepsilon_{\mathrm{eff}}-0.258\right)} \frac{W / h+0.264}{W / h+0.8},
$$

where all the dimensional parameters used above are defined in Figure 1. $\varepsilon_{r}$ and $\varepsilon_{\text {eff }}$ are the relative and effective dielectric constants, respectively.

For a matched antenna, the change in the fractional resonant frequency relative to the unloaded case or can be called as resonant frequency shift can be calculated using the following expression:

$$
\frac{\Delta f_{r}}{f_{r}}=\frac{f_{r}(d=0)-f_{r}(d)}{f_{r}(d=0)} .
$$

$f_{r}(d=0)$ can be defined as resonant frequency for unloaded sensor whilst $f_{r}(d)$ is the resonant frequency of loaded sensor with sample. The first-order change in the resonant frequency may be expressed as

$$
\frac{\Delta f_{r}}{f_{r}}=\frac{\sqrt{\varepsilon_{\mathrm{eff}}}-\sqrt{\varepsilon_{\mathrm{eff0}}}}{\sqrt{\varepsilon_{\mathrm{eff} 0}}},
$$

where $\varepsilon_{\text {effo }}$ and $\varepsilon_{\text {eff }}$ are the effective dielectric constant without sample and with sample, respectively.

The effective permittivity can be written in terms of the filling fraction, $q$, occupied by each dielectric as

$$
\varepsilon_{\mathrm{eff}}=q_{1} \varepsilon_{r 1}+q_{2} \varepsilon_{r 2}
$$

2.1.2. Finite Element Method Using COMSOL. The input values of the dielectric constant and loss factor for the samples of different percentages m.c. required in microwave office and COMSOL were obtained from the Agilent Dielectric Probe Kit $85070 \mathrm{E}$.

The finite element method to calculate reflection coefficient using COMSOL was accomplished by defining [7]. Consider

$$
\Gamma=\frac{\sqrt{P_{\text {ref }}}}{\sqrt{P_{\text {in }}}},
$$


TABLE 1: Calculation of the reflection coefficient of the unloaded sensor using COMSOL with various mesh options.

\begin{tabular}{lccc}
\hline $\begin{array}{l}\text { Types of } \\
\text { mesh }\end{array}$ & $\begin{array}{c}\text { Default } \\
\text { elements }\end{array}$ & $\begin{array}{c}\text { Number of } \\
\text { degrees of } \\
\text { freedom }\end{array}$ & $\begin{array}{c}\text { Solution } \\
\text { time }\end{array}$ \\
\hline $\begin{array}{l}\text { Extremely } \\
\text { coarse }\end{array}$ & 1069 & 7866 & $134.163 \mathrm{~s}$ \\
$\begin{array}{l}\text { Extra coarse } \\
\text { Coarser }\end{array}$ & 1411 & 10322 & $178.922 \mathrm{~s}$ \\
Coarse & 2826 & 20308 & $414.394 \mathrm{~s}$ \\
Normal & 4337 & 30598 & $865.634 \mathrm{~s}$ \\
\hline
\end{tabular}

where $P_{\text {ref }}$ and $P_{\text {in }}$ are the reflected and input powers that are given by

$$
\begin{gathered}
P_{\text {ref }}=\sqrt{P_{\text {in }}}-\sqrt{P_{\text {dissipated }}}, \\
P_{\text {in }}=\frac{1}{2} \sqrt{\left(\frac{\varepsilon_{\text {coax }}}{Z_{0}}\right)} \cdot \frac{\left(E_{r 1} \cdot j E_{r 1}\right)}{r^{2}} .
\end{gathered}
$$

$P_{\text {dissipated }}$ is the dissipated power that can be define as

$$
P_{\text {dissipated }}=\frac{V^{2}}{2\left|Z_{0}+Z_{s}\right|^{2}} \operatorname{Real}\left(Z_{s}\right) .
$$

In (11), $r$ is radius of the port, $\varepsilon_{\text {coax }}$ is the permittivity of coaxial cable, $E_{r_{1}}$ is the radial electric field of the feeding cable in unit $\mathrm{V} / \mathrm{m}, Z_{s}$ is the input impedance of the loaded sensor, $V$ is the peak value of the source, and $Z_{0}$ is the $50 \Omega$ characteristic impedance of the cable.

The boundary conditions of the domains (labeled 1 to 8) shown in Figure 3(a) were set as follows: copper (perfect electric conductor), interface between the microstrip substrate and Teflon of the feeding section (continuity), unloaded sensor (scattering boundary condition) and sensor loaded with sample (matched boundary condition) and Port is defined at the coaxial feeder. The boundary for the sample was chosen as matched boundary represented by the numbers 4, 5, 7, 9, and 69 in Figure 3(b).

Generally the higher the number of FEM mesh elements the more accurate shall be the computational result using FEM. Finer mesh will increase the number of degrees of freedom solved which in turn results in higher number of initial mesh elements for the patch sensor.

Table 1 provides the listing of the number of mesh elements together with the degrees of freedom and the processing time taken to calculate the reflection coefficient of the unloaded sensor in the frequency range from $1 \mathrm{GHz}$ to $4 \mathrm{GHz}$ for 101 points using various COMSOL mesh options.

The results for the variation in magnitude, $|\Gamma|$ of reflection coefficient with the frequency of the unloaded sensor using the various mesh elements together with experimental result are shown in Figure 4 . The resonance frequency calculated using COMSOL extremely coarse, extra coarse, coarser, and coarse options were $3.2 \mathrm{GHz}$. The coarse option calculated exactly the same value of resonance frequency measured using a vector network analyzer at $3.2 \mathrm{GHz}$ which is also almost to the value obtained using the variational technique $(3.3 \mathrm{GHz})$ but it expense of higher computational time and other computer resources.

\subsubsection{Method of Moment Implementation Using Microwave} Office. The characteristic impedance of the covered microstrip is defined as

$$
Z_{m n}=-\int_{n} E^{m} \cdot J_{n} d s
$$

where $E^{m}$ is the field of the $m$ th test mode radiating in free space, and the integral in (16) is over the surface of the $n$ the expansion mode [8].

A matrix equation is solved to get the current on each mesh. Using the method of moments, with test modes chosen identical to expansion modes, the $I_{n}$ are found as solution of the matrix equation

$$
[Z] I=V,
$$

where $[Z]$ is the symmetric $N \times N$ impedance matrix, $I$ is the current vector containing the $N$ unknown complex coefficients from (18) that the surface current on the patches is expanded as

$$
J_{s}=\sum_{n=1}^{N} I_{n} J_{n},
$$

where $J_{s}$ is the $n$th surface patch antenna mode, $N$ is the number of modes in the expansion, and $I_{n}$ is the unknown complex coefficient for the $n$th mode.

In this work, the electromagnetic simulator EMSight of the microwave office design suite from applied wave research (AWR) was used to determine the impedance of the patch sensor. A set of matrix equations is solved to get the current on each mesh. The currents are excited by attaching port, and the reflection coefficient can then be calculated. The domain conditions for the top and bottom surfaces of the enclosure were set to permittivity of the samples and 6.15 for the substrate, respectively. The entire patch antenna was subdivided into the grid by setting the number of division cell sizes of $0.1 \mathrm{~mm}$ for both of the $X$ and $Y$ directions. Smaller cell sizes provide a more accurate simulation. However if the cells are too small, the processing time would be too long or could even result in memory crashing and hang the personal computer (PC) system.

2.2. Dielectric Mixture Model. The complex permittivity values are required as input to calculate the reflection coefficient of Hevea latex using the microstrip patch sensor. The open ended coaxial probe is the most commonly used technique to determine the permittivity of samples. The permittivity is calculated from the admittance equation of the probe in conjunction with an optimized Debye model [9]. Various forms of dielectric model are also available in the literature where the dielectric mixture model is the most popularly used for heterogeneous samples such as Hevea latex. 


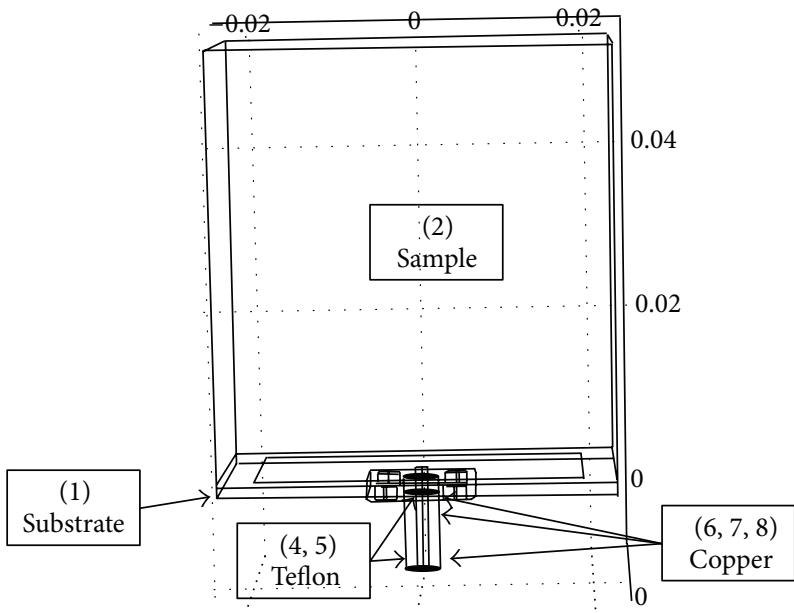

(a)

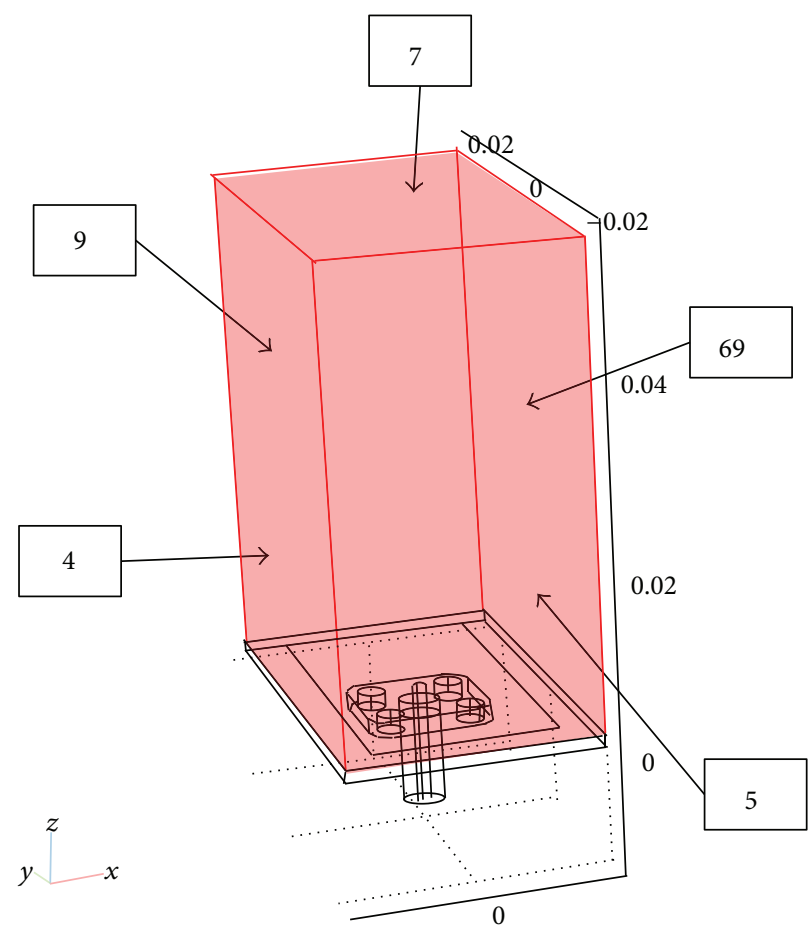

(b)

FIGURE 3: (a) Subdomain and (b) boundary for the sample.

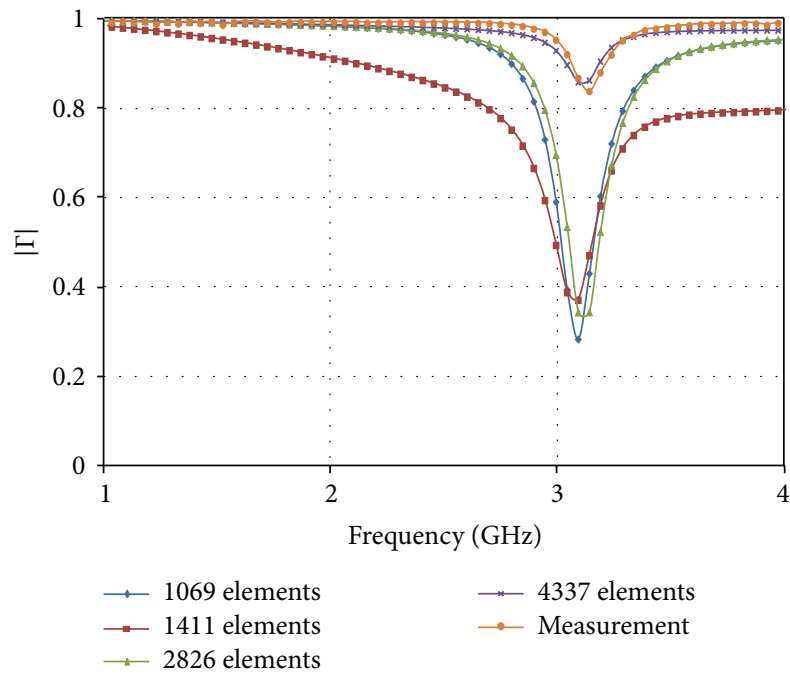

FigURE 4: Relationship between magnitude, $|\Gamma|$ of reflection coefficient and frequency of the unloaded sensor for variety of number of elements using FEM and measurement.

Previous researchers [10-13] used dielectric mixture model to predict the permittivity of materials having more than two components. The most popular dielectric mixture models are the Bruggemann, Weiner and Krazewski models [13]. However, Kraszewksi's model is still among the most commonly used model due to its simplicity requiring only the values of the volume fraction and permittivity of the each component [14]. The Kraszewski model is actually a simplified version of Wiener's model where mixtures like Hevea latex are treated as a biphase liquid, consisting of water and solid rubber. In Wiener's upper bound formula the relative dielectric permittivity of the mixture is written as

$$
\varepsilon^{*}=V_{1} \varepsilon_{1}+V_{2} \varepsilon_{2},
$$

where $\varepsilon_{1}$ and $\varepsilon_{2}$ are the relative dielectric permittivity for water and solid materials, respectively, and $V$ is the water volume fraction. Kraszewski et al. [13] derived a simplified version of Wiener's model in the from

$$
\sqrt{\varepsilon^{*}}=v_{1} \sqrt{\varepsilon_{1}}+v_{2} \sqrt{\varepsilon_{2}} .
$$

The volume fraction $V$ is related to the m.c. (wet basis) by

$$
V=M_{w 1}\left[M_{w 1}+\left(\frac{D_{2}}{D_{1}}\right)\right] .
$$

$M_{w}$ is the m.c. and $D_{1}$ and $D_{2}$ are the relative density of the water and solid rubber, respectively, and are considered to be constant with $D_{1}=1.0$ and $D_{2}=0.04$ [14]. The permittivity of latex sample can be estimated using (2) and (3).

\section{Experimental Section}

Fresh Hevea latex samples were obtained from the Universiti Putra Malaysia's Research Park. The moisture content of the fresh latex was approximately $42 \%$. The samples were diluted with different volumes of deionized water to obtain 


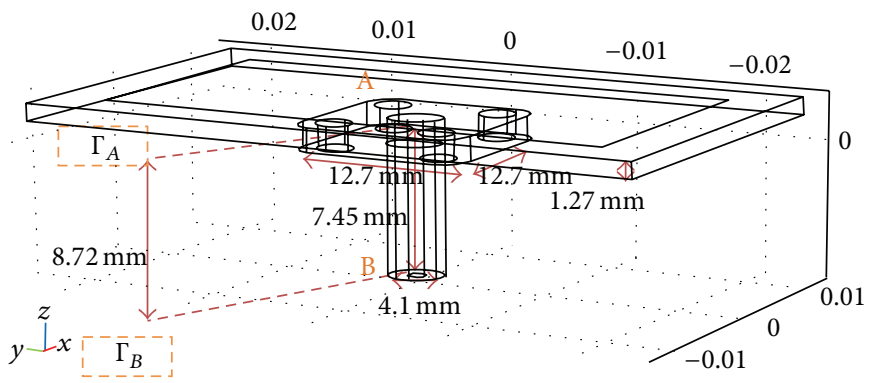

FIgURE 5: Transmission line of aperture of patch antenna.

different percentages of moisture content in latex between $35 \%$ and $85 \%$. The mass of fresh and diluted latex samples was recorded using electronic balance with a $\pm 0.1 \mathrm{mg}$ precision. The samples were dried in an oven at $70^{\circ} \mathrm{C}$ for 18 hours and six hours further drying at $105^{\circ} \mathrm{C}$ [15]. The dried samples were allowed to cool at room temperature $25^{\circ} \mathrm{C}$ before weighing. The process was repeated until a constant mass with $\pm 0.5 \mathrm{mg}$ was obtained for each sample. The actual moisture content was determined using standard oven drying method [16]. Consider

$$
\text { Moisture content }(\%)=\frac{m_{\text {wet }}-m_{\mathrm{dry}}}{m_{\text {wet }}} \times 100 \% \text {, }
$$

where $m_{\text {wet }}$ and $m_{\text {dry }}$ are the initial and final mass before and after drying. The permittivities of all the samples with different percentages of m.c. were measured using the Agilent N54030 open ended coaxial probe.

The microstrip patch sensor was fabricated using a $1.27 \mathrm{~mm}$ thick RT/Duroid 6006 substrate with a permittivity of 6.15. Ideally, the sensing area, that is, the patch dimensions must be as large as possible. However considering other factors such as rigidity and cost, the dimension $40 \mathrm{~mm}$ by $20 \mathrm{~mm}$ was selected. The theoretical resonant frequency calculated using FEM was $3.2 \mathrm{GHz}$.

The coaxial feeder of the patch antenna was a SMA stub contact panel with inner and outer conductors $1.3 \mathrm{~mm}$ and $4.1 \mathrm{~mm}$, respectively. All the calibration and reflection coefficient measurements were carried out using an Agilent N5230A professional network analyzer (PNA) in the frequency range between $1 \mathrm{GHz}$ and $4 \mathrm{GHz}$. The full oneport calibration procedure was performed at $B$ in Figures 5 and 6 using N4691-60004 $(300 \mathrm{kHz}-26.5 \mathrm{GHz})$ electronic calibration module. The reflection coefficient values of the sensor at $A$ were realized by applying the PNAs port extension technique $[17,18]$.

\section{Results and Discussions}

4.1. Permittivity of Hevea Rubber Latex. Figure 7 shows the variation in complex permittivity of water and Hevea rubber latex samples with moisture content between $36.1 \%$ and $88.6 \%$ in the frequency range from $1 \mathrm{GHz}$ to $4 \mathrm{GHz}$. It can be clearly seen from Figure 7(a) that dielectric constant of all Hevea latex samples decrease almost linearly with frequency. This suggests that the dielectric constant of Hevea latex

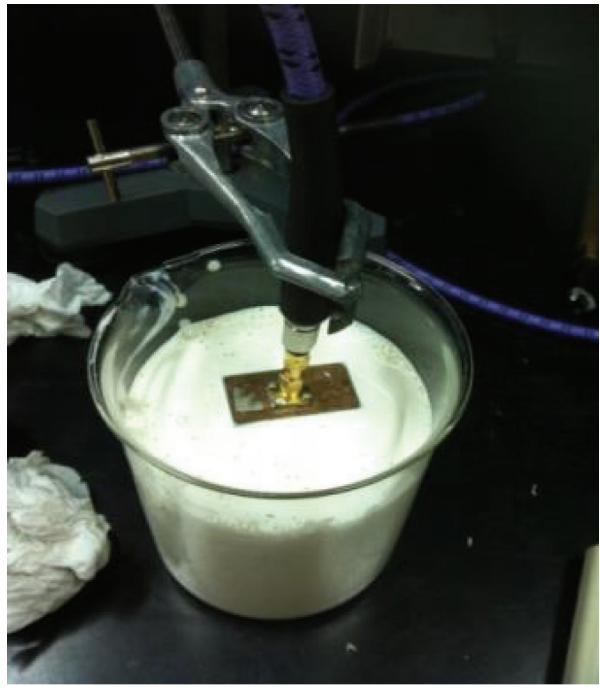

FIgURE 6: Microstrip measurement Hevea latex sample.

samples with $36.1 \%$ and $88.6 \%$ moisture content follow closely the profile of the dielectric constant of water. The higher the moisture content, the higher the dielectric constant as shown in Figure 7(a). This was expected, since water is a strong polar solvent [10]. The dielectric constant which is usually defined as the ability to store energy is lower at higher frequencies for water and latex samples. This is as expected from the Debye relationship described by Nyfors and Vainikainen, 1989 [19]. The electric polarization of water is highly influenced by the operating frequency. Water molecules were able to follow the vibration of the microwave at low frequency, thus decreasing the value of dielectric constant. Contrarily, the molecules were no longer able to follow the vibration of microwave at the high frequency and energy is dissipated as heat resulting in lower values of dielectric constant [14] but higher loss factor as shown in Figure 7(b).

The variation in the loss factor with frequency is shown in Figure 7(b). Similar to the dielectric constant, the profiles of the loss factor for all latex samples follow closely the profile of water in the frequency range from $1 \mathrm{GHz}$ to $4 \mathrm{GHz}$. The loss factor influences both energy absorption and reflection and describes the ability to dissipate energy in response to an applied electric field or various polarization mechanisms, which commonly results in heat generation $[11,20]$. The loss 


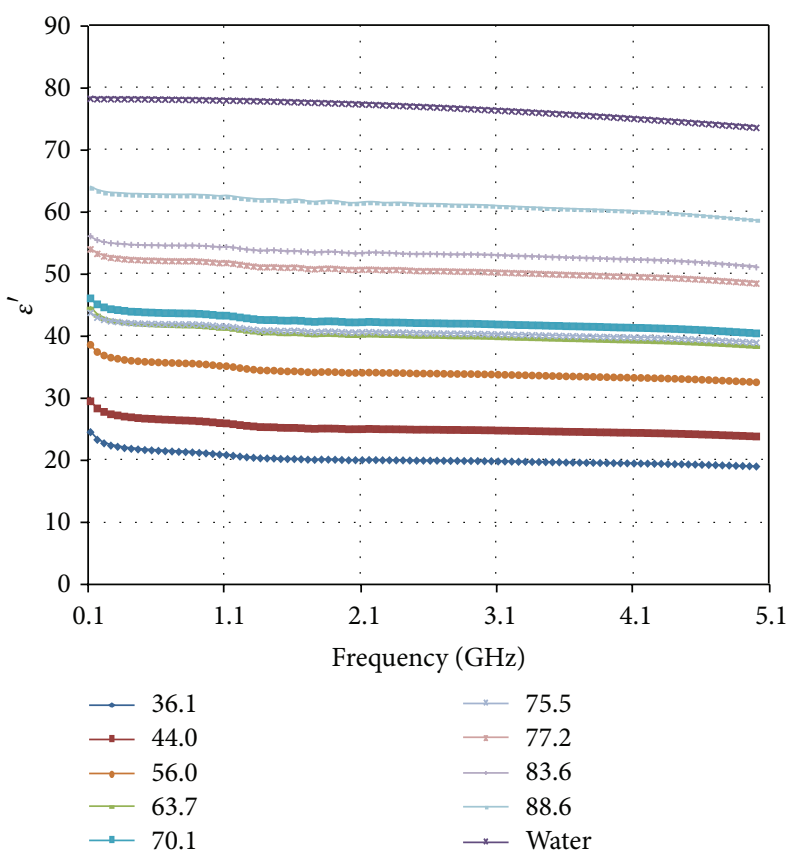

(a) Dielectric constant, $\varepsilon^{\prime}$

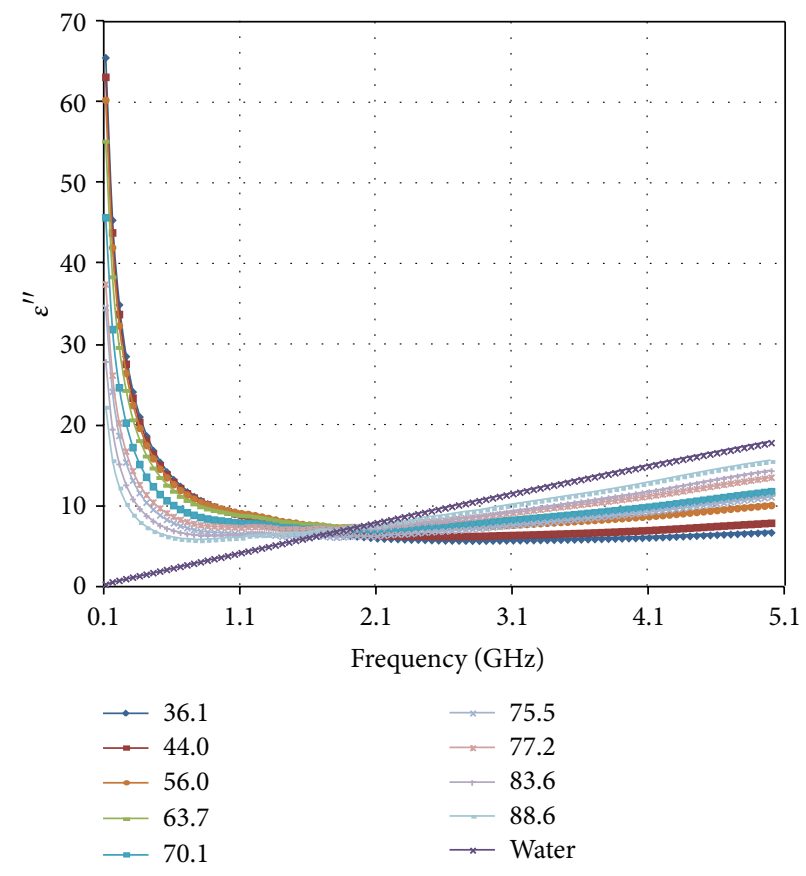

(b) Loss factor, $\varepsilon^{\prime \prime}$

Figure 7: (a) Dielectric constant and (b) loss factor of Hevea rubber latex at various percentages of m.c.

mechanisms are predominantly due to ionic conductivity and dipole rotation activities. Mechanisms that contribute to the dielectric loss factor include dipole, electronic, ionic, and Maxwell-Wagner responses [21].

The loss factor decreases as frequency increases from $0.1 \mathrm{GHz}$, reaches a broad minimum in the region between 1.5 and $2.0 \mathrm{GHz}$, and then increases as frequency approaches $4 \mathrm{GHz}$. A relaxation effect is usually associated with orientation polarization. Water has a strong dipolar effect at low frequencies. The loss mechanism is mainly influenced by ionic conduction by bound water relaxation below $2.0 \mathrm{GHz}$. Above 2.0 GHz, the dipole orientation is the dominant mechanism as the frequency approaches free water relaxation frequency [22].

4.2. Magnitude of Reflection Coefficient of Unloaded Sensor with Frequency. The comparison between the measured and calculated magnitude, $|\Gamma|$ of reflection coefficient of the unloaded sensor with frequency is illustrated in Figure 8. The calculated results were obtained from the finite element method (FEM), method of moment (MoM), and variational technique. The error distribution graph strongly suggests that the highest error between the measured and calculated $|\Gamma|$ reflection coefficient occurred at the resonant frequencies.

Both the measured and calculated FEM calculations of the fundamental resonant frequency and magnitude of reflection coefficient were found to be similar, that is, $f_{0}=$ $3.2 \mathrm{GHz}$ with $|\Gamma|=0.85$ and 0.86 , respectively. In contrast, the calculated fundamental resonant frequencies for MoM and variational technique were $3.3 \mathrm{GHz}$ with magnitudes 0.91 and 0.93 , respectively. However it should be noted that the reference plane for FEM calculation and measurement of the reflection coefficient were at the interface between the cable and the microstrip input connector whilst the MoM and variational technique were at the sensing patch and air interface. The microwave office software used to implement the MoM calculation does not have an option for feeder length of the coaxial feed when using its "Via port" object. Applying the phase extension technique at the interface between the patch and air, the measured and calculated magnitudes of reflection coefficient using FEM were 0.91 and 0.92 , which were close to the calculated MoM and variational technique.

The measured and FEM calculated $|\Gamma|$ profile using the COMSOL's coarse option is almost similar at the vicinity of the fundamental resonant frequency in the frequency range between $2.1 \mathrm{GHz}$ and $3.3 \mathrm{GHz}$ with a mean error 0.014 . The accuracy of the calculated electromagnetic field using FEM varies as functions of position and direction if the numbers of elements are too small. Thus, it is expected that higher accuracy can be obtained by using higher number of equilateral tetrahedral elements. The effect of the number of elements is discussed in Section 2.1.2. Reduced errors can be expected by using higher number of elements by choosing normal and fine with 15159 and 24240 numbers of elements, respectively. Unfortunately, these higher element options require a high performance computing facility not found in a normal PC. Continuing the use of normal and fine options would crash the PC system memory used in this work.

4.3. Effect of Moisture Content on the Resonant Frequency of the Loaded Sensor. Figure 9 shows both measured and numerical methods results for the variation in the shift 


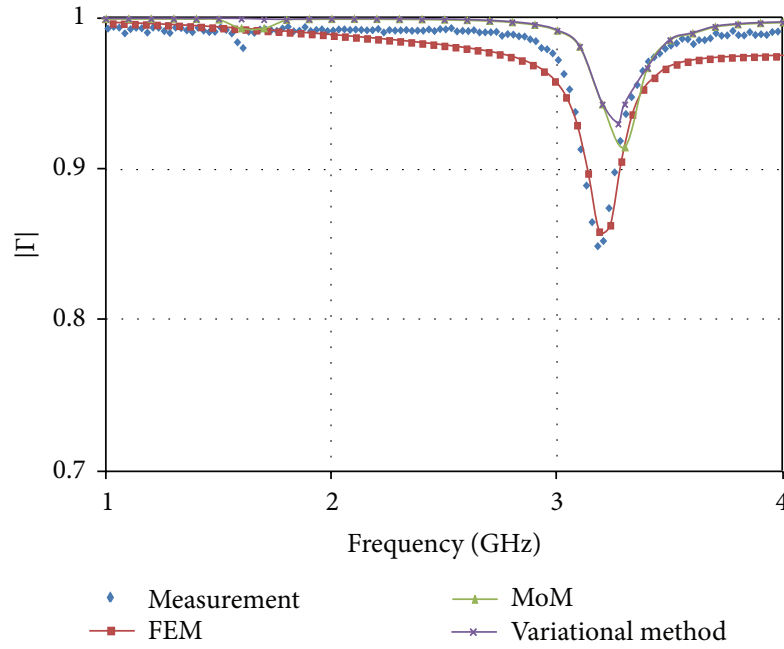

(a)

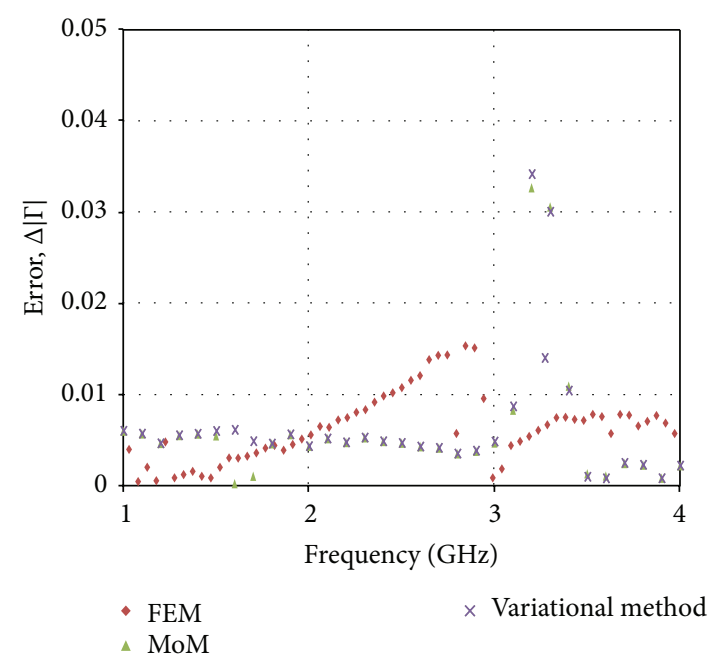

(b)

FIGURE 8: Variation in (a) magnitude, $|\Gamma|$ and (b) error magnitude, $\Delta \Gamma=\left|\Gamma_{\text {measured }}-\Gamma_{\text {calculated }}\right|$ of reflection coefficient of unloaded sensor with frequency.

TABLE 2: Relationship between resonant frequency shift, $f_{\text {shift }}$ and moisture content.

\begin{tabular}{lccc}
\hline Method & Regression equation & Regression coefficient, $R^{2}$ & Sensitivity, $(\mathrm{GHz} / \%$ m.c $)$ \\
\hline Measurement & $f_{\text {shift }}=6.62 \times 10^{6}$ m.c. $+1.59 \times 10^{8}$ & 0.7985 & 0.007 \\
FEM & $f_{\text {shift }}=1.97 \times 10^{6}$ m.c. $+4.26 \times 10^{8}$ & 0.4766 & 0.002 \\
MoM & $f_{\text {shift }}=1.64 \times 10^{7}$ m.c. $+3.77 \times 10^{8}$ & 0.9368 & 0.02 \\
Variational A & $f_{\text {shift }}=9.43 \times 10^{6}$ m.c. $-1.81 \times 10^{8}$ & 0.9999 & 0.009 \\
Variational B & $f_{\text {shift }}=7.13 \times 10^{6}$ m.c. $+8.60 \times 10^{7}$ & 0.9549 & 0.007 \\
\hline
\end{tabular}

in the resonant frequency $f_{\text {shift }}$ of the sensor loaded with samples of different percentages (\%) of m.c. It can be clearly observed that $f_{\text {shift }}$ increases almost linearly with m.c. for all the methods. The relationships are listed in Table 2. Except for variational A method utilizing the dielectric mixture model (2), the input permittivity values required in the calculation of $f_{\text {shift }}$ were obtained from Agilent HP 85070E. The measurement sensitivity, that is, the change in $f_{\text {shift }}$ with respect to the change in $m . c$ was best predicted by the variational $\mathrm{B}$ method, that is, $0.007 \mathrm{GHz} / \%$ m.c. For every $1 \%$ change in moisture content can be represented by a corresponding $0.007 \mathrm{GHz}$ change in the frequency resonance shift, $f_{\text {shift }}$ of the sensor.

The error distribution of the calculated $\Delta f_{\text {shift }}$ between the different computational methods and the measurement regression line is shown in Figure 10. The error increased with higher values of m.c. except for variational A method. This suggests improved accuracy of dielectric mixture model for samples with high moisture content values used in variational $B$ method. The mean errors for variational $B$, variational $A$, MoM, and FEM were $0.105,0.229,0.752$, and 0.1 , respectively. MoM recorded the highest mean error probably due to lack of computational accuracy when the 2nd layer is a lossy, high dielectric constant material. The FEMs low error comparable to variational B method was due to the small errors for m.c.

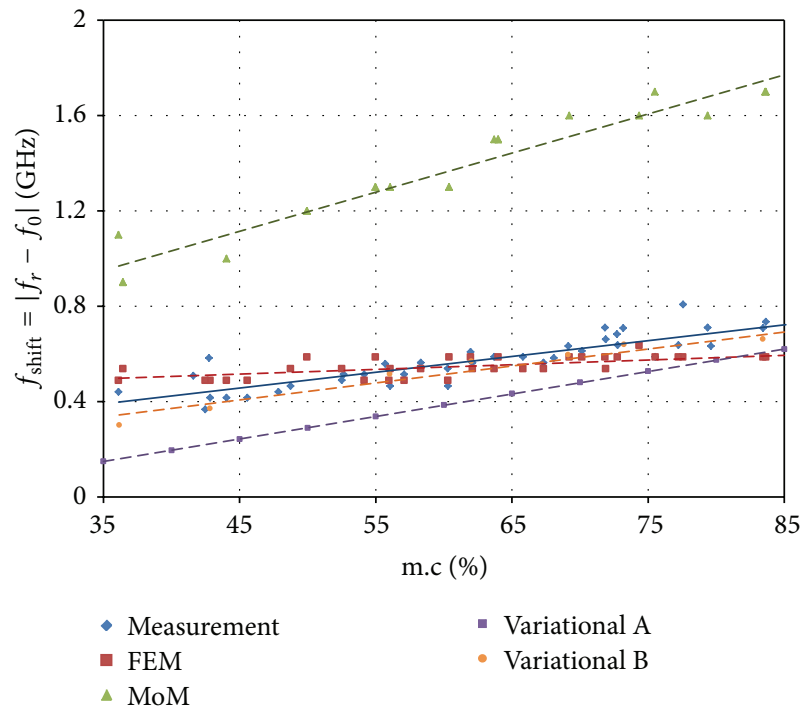

FIGURE 9: Variation in resonant frequency shift $f_{\text {shift }}=\left|f_{r}-f_{0}\right|$ with moisture content, $m$.c. (\%).

less than $55 \%$ probably to higher accuracy of the method for low permittivity samples. 


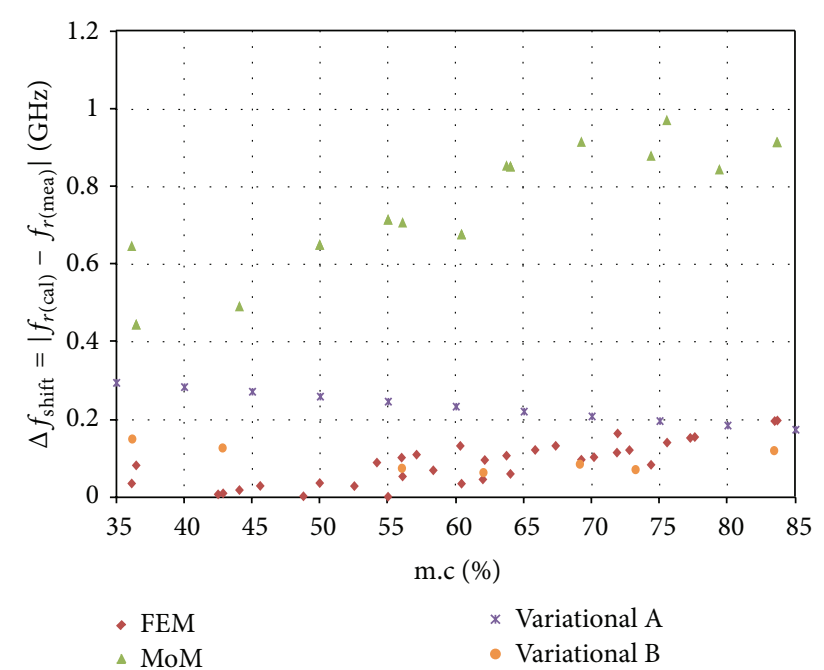

FIgURE 10: Error between measured and calculated resonant frequency shift, $\Delta f_{\text {shift }}$.

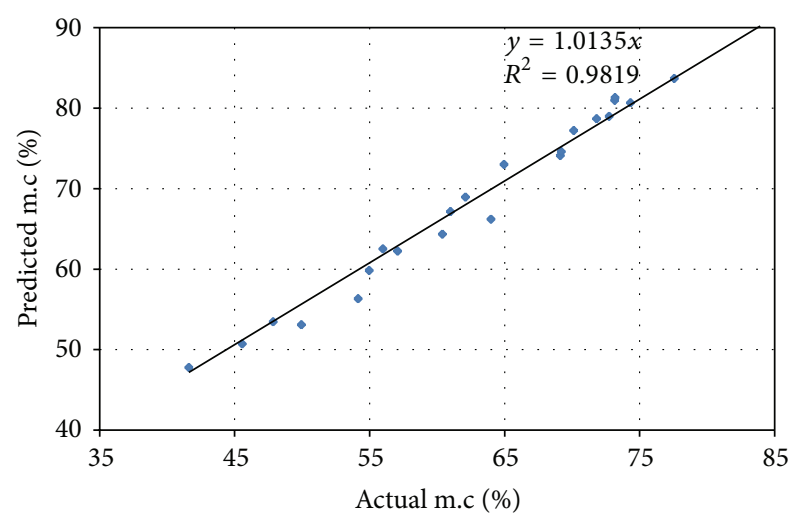

FIGURE 11: Comparison between predicted and actual m.c.

4.4. Calibration Equations to Predict Moisture Content. Calibration equations were obtained from the relationship between $f_{\text {shift }}|\Gamma|$ and $m$.c. for all the frequency points from $1 \mathrm{GHz}$ to $4 \mathrm{GHz}$. Outliers were removed using a MATLAB curve fitting toolbox. An empirical equation relating m.c. to $f_{\text {shift }}$ can be found by exchanging the $x$ and $y$-axes of Figure 9:

$$
\text { m.c. }=14.9 f_{\text {shift }}^{2}+981 f_{\text {shift }}+2.807 \text {. }
$$

Similarly from $|\Gamma|$ measurement [3]:

$$
\text { m.c. }=1177.2|\Gamma|^{2}-1554.9|\Gamma|+539.55 .
$$

The accuracy of the calibration equations was determined by calculating the errors between the actual m.c obtained from oven drying method and predicted m.c. using (20) and (21) based on $f_{\text {shift }}$ and $|\Gamma|$ measurements of a new set of latex samples. The accuracy of the predicted m.c. based on (20) and (21) was $2 \%$ and $6 \%$, respectively. The results for (20) are shown in Figure 11 where each data point represents an average of triplicates measurement.

\section{Conclusion}

The variation in resonant frequency shift, $f_{\text {shift }}$ of the microstrip sensor loaded with samples of different percentages of m.c. has been compared between measured and theoretical values using the variational method, finite element method and method of moment. Only the FEM is able to find a reasonable result, but it is not possible to perform finer meshes to improve the presented result without the support of a high performance computing facility. Calibration equation was established to predict $m$.c. from the measured $f_{\text {shift }}$ and was found to be accurate within $2 \%$ when compared to actual m.c. obtained from standard oven drying method.

\section{Conflict of Interests}

The authors declare that there is no conflict of interests regarding the publication of this paper.

\section{Acknowledgment}

The authors acknowledge financial assistance for this research from Ministry of Science, Technology and Innovation (MOSTI) for National Science Fellowship (NSF).

\section{References}

[1] K. Khalid, J. Hassan, Z. Abbas, and M. Hamami, "Microwave dielectric properties of hevea rubber latex, oil palm fruit and timber and their application for quality assessment," in Electromagnetic Aquametry: Electromagnetic Wave Interaction with Water and Moist Substances, pp. 467-490, Springer, Berlin, Germany, 2005.

[2] T. Jayanthy, "Measurement of dry rubber content in Latex using microwave technique," Measurement Science Review, vol. 5, no. 3, 2005.

[3] N. Z. Yahaya, Z. Abbas, M. A. Ismail, and B. Mohd Ali, "Determination of moisture content of hevea rubber latex using a microstrip patch antenna," in Progress in Electromagnetics Research Symposium (PIERS '12), pp. 1290-1293, Kuala Lumpur, Malaysia, March 2012.

[4] D. C. Kulkarni and V. Puri, "Perturbation of EMC microstrip patch antenna for permittivity and permeability measurements," Progress in Electromagnetics Research Letters, vol. 8, pp. 63-72, 2009.

[5] I. J. Bahl and S. S. Stuchly, "Analysis of a microstrip covered with a lossy dielectric," IEEE Transactions on Microwave Theory and Techniques, vol. 28, no. 2, pp. 104-109, 1980.

[6] E. Yamashita and R. Mittra, "Variational method for the analysis of microstrip lines," IEEE Transactions on Microwave Theory and Techniques, vol. 16, no. 4, pp. 251-256, 1968.

[7] Comsol Multiphysics, RF Module Model Library Manual Version 3.3, Comsol AB, 2006.

[8] E. H. Newman and P. Tulyathan, "Analysis of microstrip antennas using moment methods," IEEE Transactions on Antennas and Propagation, vol. 29, no. 1, pp. 47-53, 1981.

[9] D. V. Blackham and R. D. Pollard, "An improved technique for permittivity measurements using a coaxial probe," IEEE Transactions on Instrumentation and Measurement, vol. 46, no. 5, pp. 1093-1099, 1997. 
[10] S. Ryynänen, "The electromagnetic properties of food materials: a review of the basic principles," Journal of Food Engineering, vol. 26, no. 4, pp. 409-429, 1995.

[11] J. N. Ikediala, J. Tang, S. R. Drake, and L. G. Neven, "Dielectric properties of apple cultivars and codling moth larvae," Transactions of the American Society of Agricultural Engineers, vol. 43, no. 5, pp. 1175-1184, 2000.

[12] S. O. Nelson, "Dielectric properties of agricultural products and some applications," Research in Agricultural Engineering, vol. 54, no. 2, pp. 104-112, 2008.

[13] A. Kraszewski, S. Kulinski, and M. Matuszewski, "Dielectric properties and a model of biphase water suspension at $9.4 \mathrm{GHz}$," Journal of Applied Physics, vol. 47, no. 4, pp. 1275-1277, 1976.

[14] K. Kupfer, Electromagnetic Aquametry: Electromagnetic Wave Interaction with Water and Moist Substances, Springer, Berlin, Germany, 2005.

[15] "Methods of sampling and testing field latex (First Revision)," Malaysian Standard MS 466, Standard \& Research Institute of Malaysia (SIRIM), 1987.

[16] H. C. Chin, "Method of measuring the dry rubber content of field latex," in RRIM Training Manual on Analytical Chemistry, vol. 63, Rubber Research Institute Malaysia, Kuala Lumpur, Malaysia, 1979.

[17] Agilent Technologies Inc, Agilent Basics of Measuring the Dielectric Properties of Materials, Application Note, Agilent Technologies Inc, Santa Clara, Calif, USA, 2005.

[18] Y. K. Yeow, Z. Abbas, and K. Khalid, "Application of microwave moisture sensor for determination of oil palm fruit ripeness," Measurement Science Review, vol. 10, no. 1, pp. 7-14, 2010.

[19] E. Nyfors and P. Vainikainen, Industrial Microwave Sensors, Artech House, Norwood, Mass, USA, 1989.

[20] R. E. Mudgett, "Electrical properties of foods," in Engineering Properties of Foods, M. A. Rao and S. S. H. Rizvi, Eds., pp. 329390, Marcel Dekker, New York, NY, USA, 1986.

[21] A. C. Metaxas and R. J. Meredith, Industrial Micro Wave Heating, Peter Peregrinus, London, UK, 1993.

[22] M. S. Venkatesh and G. S. V. Raghavan, "An overview of microwave processing and dielectric properties of agri-food materials," Biosystems Engineering, vol. 88, no. 1, pp. 1-18, 2004. 

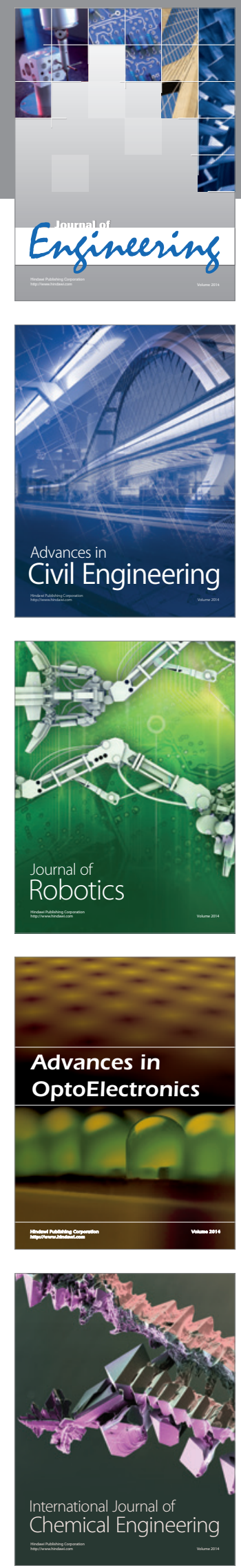

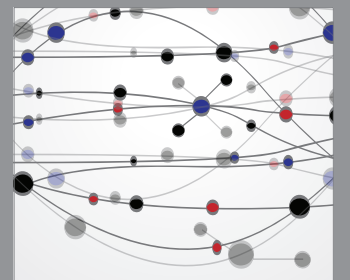

The Scientific World Journal
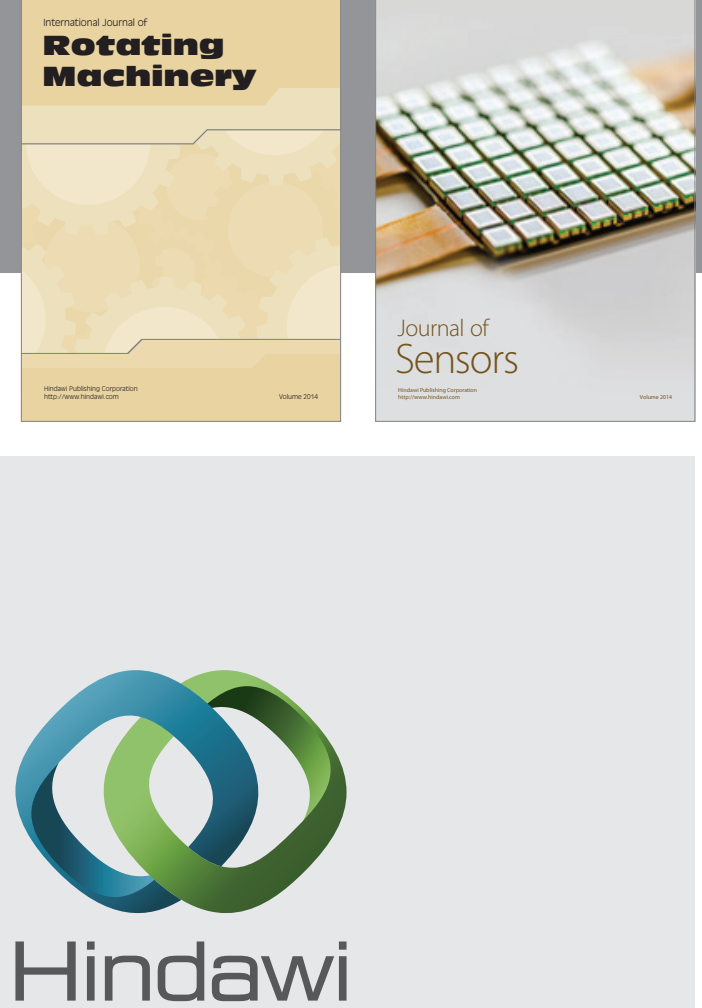

Submit your manuscripts at http://www.hindawi.com
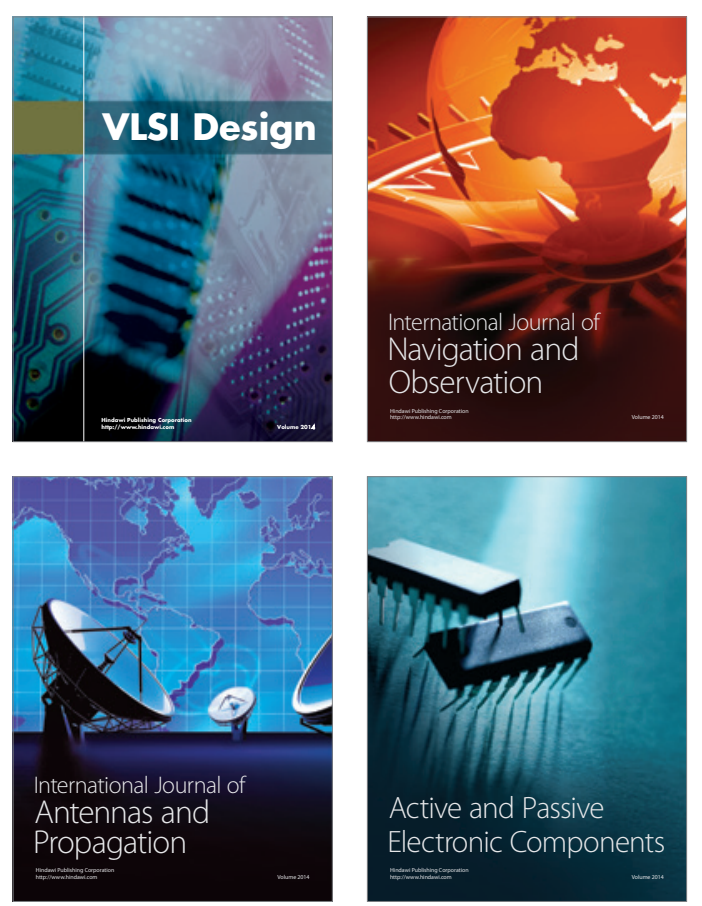
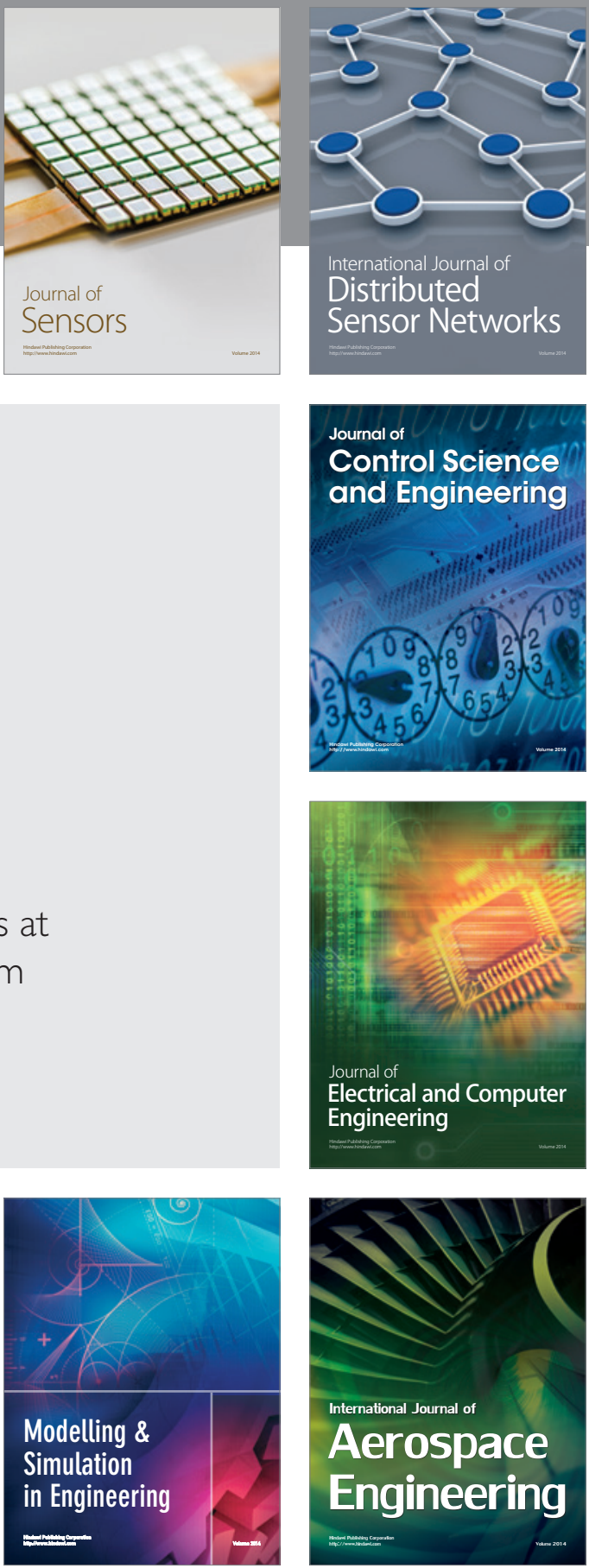

Journal of

Control Science

and Engineering
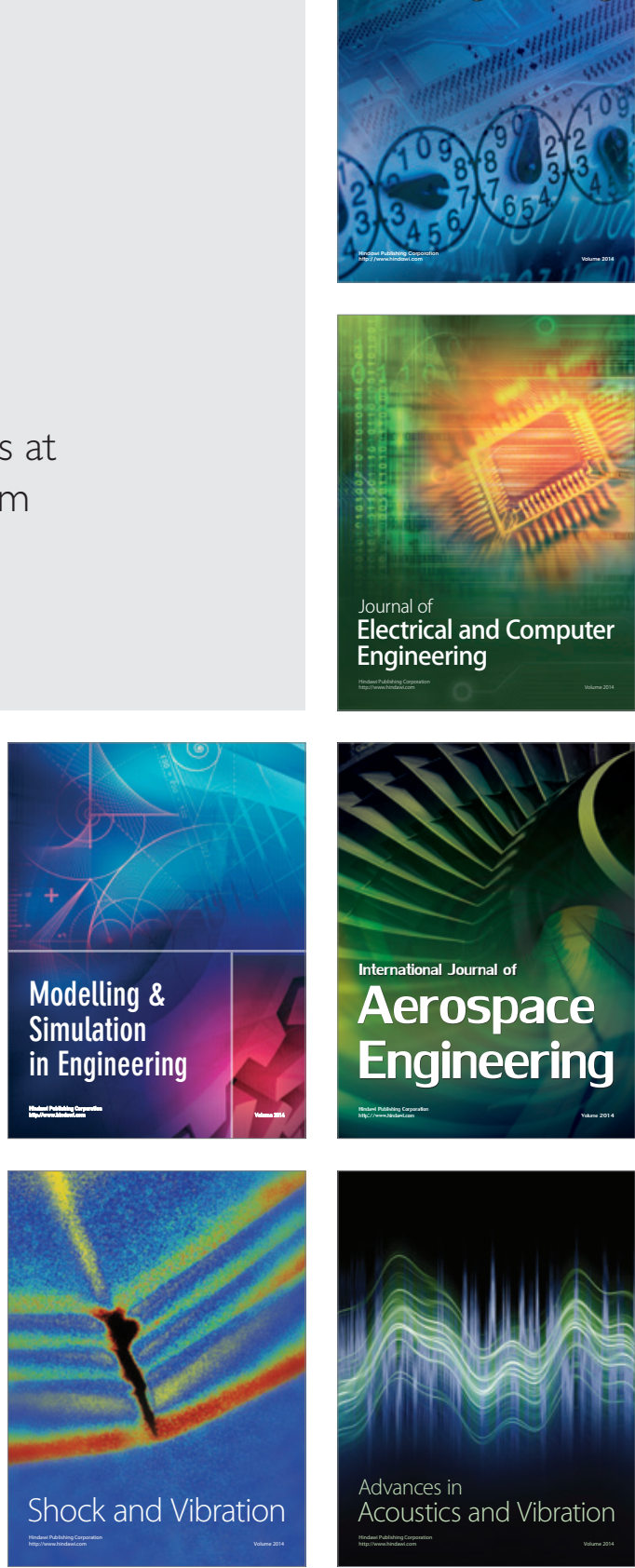